\title{
First solar butterfly diagram from Schwabe's observations in 1825-1867
}

\author{
Rainer Arlt ${ }^{1}$ and Anastasia Abdolvand ${ }^{2}$ \\ ${ }^{1}$ Astrophysikalisches Institut Potsdam, \\ An der Sternwarte 16, D-14482 Potsdam, Germany \\ email: rarlt@aip.de \\ ${ }^{2}$ Lycée Fran çais de Berlin \\ Berlin, Germany \\ email: abdolvand_a@hotmail.de
}

\begin{abstract}
The original sunspot observations by Samuel Heinrich Schwabe of 1825-1867 were digitized and a first subset of spots was measured. In this initial project, we determined more than 14000 sunspot positions and areas comprising about $11 \%$ of the total amount of spots available from that period. The resulting butterfly diagram has a typical appearance, but with evident north-south asymmetries.
\end{abstract}

Keywords. Sun: activity, sunspots, history and philosophy of astronomy

\section{Introduction}

A continuous set of sunspot positions was constructed from the observations at the Royal Greenwich Observatory starting in 1874 and the observations obtained by the USAF/NOAA starting in 1976 (cf. Hathaway et al. 2003). Numerous investigations regarding the butterfly diagram or sunspot area are based on this dataset. It is desirable to extend this information back in time to cover a larger part of the period for which sunspot numbers are known. Spörer $(1874,1878,1880,1886,1894)$ gives sunspot positions for the period 1861-1894. A short set of observations is available from Richard Carrington (1863) covering the period 1853-1861. An even earlier set of sunspot positions was derived by Arlt (2009) from the observations by Staudacher in 1749-1799.

In this paper, we present a first set of sunspot positions and areas from the full-disk drawings of the sun by Samuel Heinrich Schwabe at his location in Dessau, Germany, in 1825-1867. The original observations are preserved by the Royal Astronomical Society, London, and have been kindly provided for digitization in 2009.

\section{Determination of positions and areas}

The total number of full-disk drawings in these records is 8468 consisting of circles of $5 \mathrm{~cm}$ in diameter. Within this set, 7299 drawings have a coordinate system which is found to be aligned with the celestial equator. Especially from mid-1830 onward, almost all drawings have this coordinate system. Special care was apparently taken by Schwabe, that the drawing represents the situation at $12^{\mathrm{h}}$ local time. Schwabe gave descriptions of the spots several times a day, and in nearly all cases only the $12^{\mathrm{h}}$ description matches the drawing. All observations were made looking through a Keplerian telescope equipped 
with one of a variety of solar filters. All images have thus been turned by $180^{\circ}$ before being used for measurements.

We assume that the middle horizontal line of the drawings is parallel to the celestial equator and add the inclinations of the ecliptic as well as the tilt of the solar rotation axis against the ecliptic to the direction to the celestial pole. A preliminary heliographic coordinate system is drawn onto the sunspot drawing. The disks are enlarged to a size of 490 pixels radius to achieve a slight sub-pixel accuracy as compared to the original images. This corresponds to a scale of $0.05 \mathrm{~mm} /$ pixel which is below the probable plotting errors of Schwabe. If that was $1 \mathrm{~mm}$ (which is likely to be an upper limit), the error in heliographic position is $2.5^{\circ} / \cos d$, where $d$ is the distance from the disk center. The additional uncertainty in determining the position angle of the solar equator is included here with an approximate error of $0.3^{\circ}$; it is fairly well defined.

The cases without any coordinate system required special care. We noticed that the drawings were fairly well aligned with the celestial equator, simply by looking at the plausibility of the spot distribution from day to day. This alignment is remarkably consistent throughout the dataset, especially for the drawings explicitly marked to be made at $12^{\mathrm{h}}$ local time. We utilized those by assuming that an imaginary horizontal line through the drawing is parallel to the celestial equator. Even if one of Schwabe's early telescopes was not mounted parallactically, an alignment with the horizon in an alt-azimuth system would deliver the same result for observations near noon.

For a few observations before 1830, we used a special matching algorithm for pairs of observations separated by no more than a few days. Two or more spots need to be on both drawings. Using the differential rotation derived by Balthasar et al. (1986), the individual position angles of the two drawings can be determined by a least-squares search. While the method may deliver additional positions, not measurable otherwise, we need to bear in mind that using the differential rotation as an input reduces the chances of an independent determination of the differential rotation of that Cycle 7.

The sunspot size was estimated by various cursor masks with radii from 1 to 11 pixels. As soon as Schwabe distinguished penumbra and umbra in his drawings, we used the umbral area for the size determinations, since we believe these are a more direct indication for the emerging magnetic flux than the total size of a sunspot. Note that this differs from the USAF/NOAA set which only reports the penumbral area after Dec 16, 1981. We also need to emphasize that we only employed the full-disk drawings by Schwabe. Numerous detailed drawings of individual groups are also available, showing more spots at greater detail, but placing them to the right scale and with correct orientation into the full-disk drawings is a delicate task.

\section{Result}

The tentative butterfly diagram of the period of $1825-1867$ is shown in Fig. 1 . The darkness of the individual spots is scaled with the area of the spots while the vertical (latitudinal) extent is scaled with the diameter of the spots. Note that the diagram contains only about $11 \%$ of the full set of observations available. The accuracy will improve significantly after measuring the remaining $89 \%$ of the drawings.

The solar activity cycle is clearly visible. The first cycle (Cycle 7) appears in darker colours, because Schwabe did not distinguish the umbra from the penumbra until 1830 . The larger areas measured result in a "darker" cycle as compared to the following cycles. A recalibration of these data will be necessary for the final construction of the 


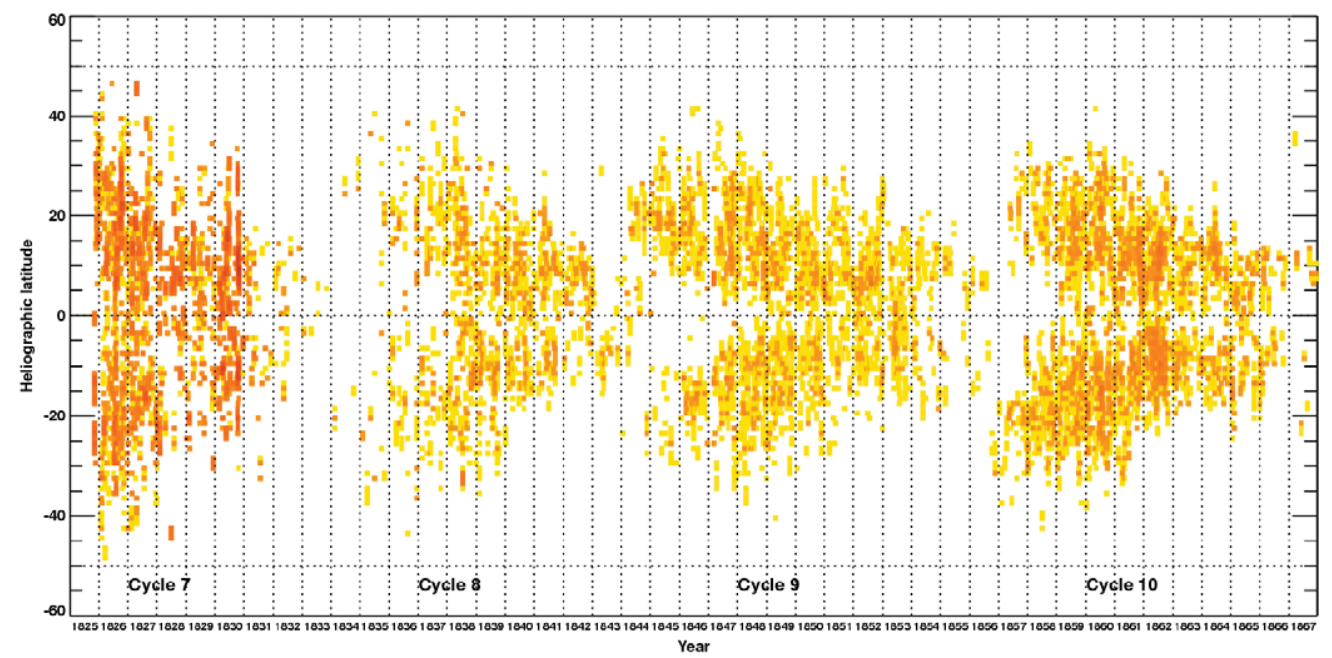

Figure 1. Butterfly diagram obtained from the observations by Heinrich Schwabe in 1825-1867. The sunspot areas up to 1830 are overestimated since Schwabe did not distinguish penumbrae from umbrae in the beginning.

butterfly diagram in the future. Also, there are some periods during Cycle 7 when we made measurements for each drawing, while other periods were covered by every 10th drawing to get a first picture of the entire observing period. These "daily" measurements are reflected as denser stripes in the butterfly diagram.

According to our results, Cycle 8 was a rather weak one, while the Wolf numbers indicate a stronger cycle. Cycle 9 shows a phase difference between the northern and southern hemispheres with the beginning of the northern cycle preceding the one of the southern hemisphere by at least half a year. The analysis as in Zolotova et al. (2010), once the full dataset is available, will show the entire development of the phase difference for the middle of the 19th century. Cycle 10 appears to be more symmetric, and the beginning of Cycle 11 is just barely visible in 1867 .

The data will be available from the author once the full set of observations will be measured.

\section{Acknowledgements}

The authors are much obliged to the Royal Astronomical Society, London, and to Robert Massey and Peter Hingley in particular for the support with digitizing the original observations. We are grateful to Stela Frencheva, Jennifer Koch, and Christian Schmiel for their help with the utilization of the digital images.

\section{References}

Arlt, R. 2009, Sol. Phys., 255, 143

Balthasar, H., Vázquez, M., \& Wöhl, H. 1986, A\& A, 155, 87

Carrington, R. C. 1863, Observations of the Spots on the Sun from November 9, 1853 to March 24, 1861 made at Redhill. Williams and Norgate, London, Edinburgh

Hathaway, D. H., Nandy, D., Wilson, R. M., \& Reichmann, E. J. 2003, ApJ, 589, 665

Spörer, G. 1874, Publicationen der Astronomischen Gesellschaft, XIII

Spörer, G. 1878, Publicationen des Astrophysikalischen Observatoriums zu Potsdam Nr. 1, Vol. 1, part 1, Wilhelm Engelmann, Leipzig 
Spörer, G. 1880, ibid Nr. 5, Vol. 2, part 1

Spörer, G. 1886, ibid Nr. 17, Vol. 4, part 4

Spörer, G. 1894, ibid Nr. 32, Vol. 10, part 1

Zolotova, N. V., Ponyavin, D. I., Arlt, R., \& Tuominen, I. 2010, AN, 331, 765 